\title{
Associations between leisure time physical activity and cardiovascular risk factors among older adults with long-term spinal cord injury
}

\author{
Sophie Jörgensen ${ }^{1,2} \cdot$ Stina Svedevall ${ }^{1} \cdot$ Linnea Magnusson ${ }^{1} \cdot$ Kathleen A. Martin Ginis $\mathbb{D}^{3} \cdot$ Jan Lexell ${ }^{1,2,4}$
}

Received: 19 October 2018 / Revised: 28 November 2018 / Accepted: 3 December 2018 / Published online: 8 January 2019

(c) International Spinal Cord Society 2019

\begin{abstract}
Study Design This is a cross-sectional study.

Objectives To describe the association between cardiovascular risk factors (body mass index (BMI), waist circumference (WC), blood pressure, blood glucose and lipids) and leisure time physical activity (LTPA) in older adults with long-term spinal cord injury (SCI).

Setting Community settings, southern Sweden.

Methods Data from the baseline data collection of the Swedish Aging with Spinal Cord Injury Study (SASCIS) ( $\mathrm{n}=123$, $71 \%$ men, injury levels C1-L5, American Spinal Injury Association Impairment Scale A-D, mean age 63 years, mean time since injury 24 years). Data were collected through home interviews, assessments and blood samples. The Physical Activity Recall Assessment for People with SCI was used to assess LTPA. Associations were investigated using multivariable linear regression analyses adjusted for age, gender, level and severity of injury, cause of injury, time since injury and tobacco use. Results More minutes per day of moderate-to-heavy LTPA were significantly associated with a lower BMI (Beta $=-0.31$; $p=0.001)$ and a lower WC (Beta $=-0.24 ; p=0.009)$. More minutes per day of total LTPA (mild intensity or greater) were associated with a higher systolic blood pressure (Beta $=0.27 ; p=0.041)$ among participants with tetraplegia. No other significant associations between the cardiovascular risk factors and total LTPA were found.

Conclusion Participation in daily LTPA is associated with better cardiovascular health with regard to BMI and WC in older adults with long-term SCI. Further studies are needed to establish the specific amount of activity needed to obtain positive health effects in this group and the directional causality of the associations.
\end{abstract}

Sponsorship Not applicable.

\section{Introduction}

Persons with spinal cord injury (SCI) are at greater cardiovascular risk compared to the general population [1-4], and $30-40 \%$ of deaths in the chronic SCI population are

Sophie Jörgensen

sophie.jorgensen@med.lu.se

Department of Health Sciences, Lund University, Lund, Sweden

2 Department of Neurology and Rehabilitation Medicine, Skåne University Hospital, Lund, Sweden

3 School of Health and Exercise Sciences, University of British Columbia, Okanagan Campus, Kelowna, BC, Canada

4 Department of Neuroscience, Rehabilitation Medicine, Uppsala University, Uppsala, Sweden caused by an underlying or contributing cardiovascular disease (CVD) [5, 6]. This elevated risk could be explained by autonomic dysfunction, metabolic changes, altered body composition and a sedentary lifestyle after injury [1, 4]. Some of these risk factors are potentially modifiable which calls for elucidation of lifestyle-related preventative measures to reduce the risk of CVD after SCI. Such measures are especially important in the long-term SCI-management, as both older chronological age and longer time since injury add to cardiovascular risk [7, 8].

In the general population, physical activity is strongly associated with beneficial effects on well-known risk factors for CVD, such as overweight, diabetes, dyslipidemia and hypertension $[9,10]$. The type of physical activity associated with health benefits is referred to as leisure time physical activity (LTPA), i.e., physical activity performed during free time, such as walking and exercising [11]. There 
is also evidence of cardiovascular benefits of moderate-toheavy intensity LTPA after SCI [12, 13], and international exercise guidelines specific to adults with chronic SCI have recently been developed [14]. These guidelines specify the type and minimum amount of exercise to achieve fitness and cardiometabolic benefits in this group [14]. However, there was insufficient evidence to draw conclusions on the health effects of LTPA in the aged SCI population $[13,14]$. This lack of evidence for older adults with chronic SCI limits the ability for scientists to formulate evidence-based exercise guidelines for all segments of the SCI population. Given the growing number of older adults living long-term with SCI, ways to promote healthy and active aging after injury is of increasing clinical and scientific interest $[4,15,16]$. There is thus a need for increased knowledge of the benefits of LTPA in this specific population.

We have, to our knowledge, for the first time, described participation in LTPA and associated factors in a Swedish sample of older adults with long-term SCI [17]. In agreement with previous population-based studies of persons with SCI of different ages [1, 18], we found that LTPA participation varied greatly, and many of the older adults did not engage in any LTPA [17]. Also, we have recently reported on a high occurrence of cardiovascular risk factors in the same Swedish SCI-sample [19]. In that study, we found only weak associations between the occurrence of risk factors and non-modifiable sociodemographics and injury characteristics [19]. Further studies are therefore needed to increase our knowledge about modifiable factors associated with the specific cardiovascular risk profile of older adults with long-term SCI.

As there are, to the best of our knowledge, no studies of the association between cardiovascular risk factors and LTPA in older adults with long-term SCI, further research is needed to establish LTPA recommendations targeting cardiovascular health in this population.

The objective of this study is to determine the association between cardiovascular risk factors (body mass index, waist circumference, blood pressure, blood lipid profile, fasting plasma glucose) and participation in LTPA in older adults with long-term SCI. We hypothesize that more minutes of LTPA are associated with lower levels of risk factors in this group $[12,20]$.

\section{Methods}

\section{Research design}

This study is based on the baseline data collection of the Swedish Aging with Spinal Cord Injury Study (SASCIS) (for details, see Jörgensen et al. [21]), a population-based, longitudinal cohort study assessing individuals 50 years or older and at least 10 years after SCI. The SASCIS is the first longitudinal study in Northern Europe aiming to contribute to the knowledge base of factors associated with healthy aging in individuals with long-term SCI [16, 17, 19, 22, 23].

\section{Participants}

All participants were living in the community and recruited through databases available at the SCI Unit at Skåne University Hospital in Lund, Sweden. The SCI Unit serves a catchment area of approximately 1.8 million people and admits 35-55 individuals with SCI for primary rehabilitation each year. At the time of recruitment (year 2011), 184 individuals met the inclusion criteria of the SASCIS (i.e., at least 50 years old and at least 10 years after traumatic SCI or non-progressive, acquired non-traumatic SCI). The final study sample comprised 123 individuals (36 women and 87 men, $67 \%$ response rate) with a mean age of 63 years (range: 50-89 years) and a mean time since injury of 24 years (range: 10-56 years). There were no significant differences between the study participants and the nonparticipants regarding gender, chronological age, time since injury, age at injury, level and severity of injury and cause of injury [21]. On the basis of the American Spinal Injury Association Impairment Scale (AIS) [24], the following three groups of SCI severity were formed: (i) tetraplegia AIS A-C $(n=22 ; 15$ AIS A, 4 AIS B and 3 AIS C); (ii) paraplegia AIS A-C $(n=41 ; 23$ AIS A, 8 AIS B and 10 AIS C); and (iii) all AIS D $(n=60)$ [21].

\section{Data collection}

In the SASCIS, the data were collected through interviews and assessments in the participants' homes $(n=122)$ or at another place $(n=1)$. Twelve (4 SCI-specific and 8 generic) internationally validated and widely used assessment tools were used together with a study-specific questionnaire (for details, see [21]); in the present study, a subset of the data were used to address the objective.

\section{Sociodemographics, injury characteristics and tobacco use}

The data on sociodemographics (gender, age), injury characteristics (age at injury, time since injury, level and severity of injury, cause of injury) and tobacco use were collected from the participants' medical records and the study-specific questionnaire.

\section{Body mass index and waist circumference}

The participants were weighed wearing light clothing and no shoes, using a portable scale for wheelchairs (Corina Medical MPWS 300; Rörvik, Sävsjö, Sweden), and $0.5 \mathrm{~kg}$ 
were subtracted from the obtained body weight. Supine height was measured using a flexible, stretch-resistant measuring tape. Body mass index (BMI) was calculated as $\mathrm{kg} / \mathrm{m}^{2}$. Waist circumference (WC) was measured in the supine position at the umbilical level after a normal expiration, using a flexible, stretch-resistant measuring tape.

\section{Blood pressure, lipid profiles and fasting plasma glucose}

Sitting blood pressure (BP) was measured manually in the right arm. Blood analyses of lipid profiles (i.e., total cholesterol (TC), triglycerides (TG), low-density lipoprotein cholesterol (LDL-c), high-density lipoprotein cholesterol (HDL-c)) and fasting plasma glucose (FPG) were obtained at the participants' local primary health care center. Analyses of blood samples were conducted according to standardized hospital laboratory methods. In case of hypertension, dyslipidemia and/or elevated FPG levels, participants were referred to his/her primary care physicians for further investigation and treatment. A detailed description of the occurrence of cardiovascular risk factors in the SASCIS population has been presented elsewhere [19].

\section{Leisure time physical activity}

LTPA was assessed using the Physical Activity Recall Assessment for People with Spinal Cord Injury (PARA$\mathrm{SCI}$ [25]. The assessment is based on self-report and performed according to a standardized, structured interview protocol [26]. The participants were asked about the type, frequency, amount and intensity of all physical activities (i.e., LTPA and activities of daily living) they had performed during the past three days. The intensity was classified using a SCI-specific chart [25]. In this study, only data on LTPA were used, presented as the mean number of minutes per day of LTPA of at least mild intensity (i.e., total LTPA) and of moderate-to-heavy intensity (i.e., moderateto-heavy LTPA), respectively. A detailed description of LTPA participation and associated factors within the SASCIS study sample has been presented elsewhere [17].

\section{Data and statistical analyses}

The data were processed using IBM SPSS Statistics for Windows v. 25 (IBM Corporation, Armonk, NY, USA). Descriptive statistics were calculated for sociodemograhics, injury characteristics, tobacco use, BMI, WC, BP, lipid profiles, FPG and LTPA. For data collected with the PARASCI, two outliers (greater than the mean \pm 3 standard deviations (SD)) were reduced to the next lowest value in the distribution. Multivariable linear regression models were computed with each of the cardiovascular risk factors as dependent variables. Separate sets of models were computed for total LTPA and moderate-to-heavy LTPA as independent variables, and all models were controlled for age, gender, level and severity of injury, cause of injury, time since injury and tobacco use (current daily smoker; yes/no). As the level and severity of injury were represented by three categories, dummy variables were created with the all AIS D as the reference category. Four participants did not complete the PARA-SCI and were excluded from the regression analyses. Only the regression models where a significant association between LTPA and cardiovascular risk factors could be found are presented in the results; these models exhibited no influential cases, no residual outliers, no heteroscedasticity, no multicollinearity and the residuals were normally distributed.

\section{Results}

\section{Sociodemographics and injury characteristics}

In Table 1, the data on the participants' sociodemographics and injury characteristics are presented. Their mean (SD) age was 63 (9) years, ranging between 50 and 89 years. The mean (SD) time since injury was 24 (12) years, the mean (SD) age at injury 39 (16) years and the majority (71\%) were men. Almost half (49\%) of the participants presented with an AIS D injury and almost two thirds (62\%) had sustained a traumatic SCI.

Table 1 Sociodemographics and injury characteristics of older adults with long-term spinal cord injury $(n=123)$

\begin{tabular}{ll}
\hline & $\mathrm{n}(\%) ;$ mean \pm SD; median, min-max \\
\hline Gender & $87(71)$ \\
$\quad$ Men & $36(29)$ \\
$\quad$ Women & $63 \pm 9 ; 63,50-89$ \\
Age (years) & $39 \pm 16 ; 38,7-74$ \\
Age at injury (years) & $24 \pm 12 ; 22,10-56$ \\
Time since injury (years) & \\
Cause of injury & $76(62)$ \\
$\quad$ Traumatic & \\
$\quad$ Non-traumatic & \\
Level and severity of injury & \\
$\quad$ Tetraplegia AIS A-C & $22(18)$ \\
Paraplegia AIS A-C & $41(33)$ \\
All AIS D & $60(49)$
\end{tabular}

AIS American Spinal Injury Association (ASIA) Impairment Scale [24]; $S D$ standard deviation

${ }^{\mathrm{a} T r a f f i c / t r a n s p o r t a t i o n, ~ f a l l, ~ w o r k p l a c e ~ a c c i d e n t, ~ d i v i n g ~ a c c i d e n t, ~}$ gunshot/assault/torture, other traumatic

${ }^{\mathrm{b}}$ Spinal tumor, spinal disc herniation, spinal arteriovenous malformation, spinal infarction, spinal infection 
Table 2 Body mass index, waist circumference, blood pressure, blood analyses, tobacco use and participation in leisure time physical activity among older adults with long-term spinal cord injury

\begin{tabular}{|c|c|}
\hline & mean $\pm \mathrm{SD} ;$ median, min-max $n(\%$ \\
\hline $\mathrm{BMI} ; \mathrm{kg} / \mathrm{m}^{2}(n=121)$ & $27.2 \pm 4.5 ; 26.6,14.1-40.2$ \\
\hline Waist circumference; $\mathrm{cm}(\mathrm{n}=119)$ & $101.2 \pm 12.9 ; 100.0,65.0-134.0$ \\
\hline Systolic blood pressure; $\mathrm{mm} \mathrm{Hg}(n=121)$ & $135.6 \pm 24.3 ; 135.0,73.0-195.0$ \\
\hline Diastolic blood pressure; $\mathrm{mm} \mathrm{Hg}(n=121)$ & $82.5 \pm 12.1 ; 84.0,54.0-110.0$ \\
\hline Total cholesterol; $\mathrm{mmol} / \mathrm{l}(n=116)$ & $4.9 \pm 1.1 ; 4.8,2.6-8.2$ \\
\hline Triglycerides; mmol/l $(n=116)$ & $1.6 \pm 1.1 ; 1.2,0.4-8.7$ \\
\hline Low-density lipoprotein cholesterol; mmol/l $(n=116)$ & $3.1 \pm 1.0 ; 3.0,1.1-6.1$ \\
\hline High-density lipoprotein cholesterol; mmol/l $(n=116)$ & $1.3 \pm 0.5 ; 1.3,0.6-3.6$ \\
\hline Fasting plasma glucose; mmol/l $(n=111)$ & $5.9 \pm 1.7 ; 5.5,3.8-14.7$ \\
\hline Current daily smoking $(n=123)$ & $20(16)$ \\
\hline Total LTPA ${ }^{\mathrm{a}} ; \min /$ day $(n=119)$ & $34.7 \pm 41.5 ; 15,0.0-171.7$ \\
\hline Moderate-to-heavy LTPA ${ }^{\mathrm{b}}$; min/day $(n=119)$ & $22.5 \pm 35.1 ; 5,0.0-140.0$ \\
\hline
\end{tabular}

\section{Cardiovascular risk factors and leisure time physical activity}

In Table 2, data on the participants' BMI, WC, BP, lipid profiles, FPG, tobacco use and participation in LTPA are presented. The participants' mean (SD) BMI was 27.2 (4.5) $\mathrm{kg} / \mathrm{m}^{2}$, and their mean (SD) WC was $101.2(12.9) \mathrm{cm}$. Sixteen percent smoked cigarettes on a daily basis. There was a large variation in reported total LTPA, with a mean (SD) of 34.7 (41.5) min per day (median 15.0 min per day). A total of $29 \%$ did not engage in any LTPA. The mean (SD) amount of moderate-to-heavy LTPA was 22.5 (35.1) (median of $5.0 \mathrm{~min}$ per day).

\section{Associations between cardiovascular risk factors and leisure time physical activity}

Moderate-to-heavy LTPA was associated with some of the risk factors and these associations are presented in Table 3. The independent variables explained between 10 and $32 \%$ of the variance in the cardiovascular risk factors. More min per day of moderate-to-heavy LTPA were significantly associated with a lower BMI (Beta $=-0.31 ; p=0.001)$ and a lower WC (Beta $=-0.24 ; p=0.009)$ (Table 3). No other significant associations between the cardiovascular risk factors and moderate-to-heavy LTPA were found.

Total LTPA was not associated with any of the cardiovascular risk factors when controlled for age, gender, level and severity of injury, cause of injury, time since injury and tobacco use; therefore, the results from these regression models are not presented. When participants with tetraplegia $(n=46)$ and paraplegia $(n=71)$ were analyzed separately, controlling for age, gender, cause of injury, time since injury and tobacco use, more minutes per day of total
LTPA were significantly associated with a higher systolic BP $($ Beta $=0.27 ; p=0.041)$ among participants with tetraplegia.

\section{Discussion}

The present study, to our knowledge, is the first to describe the association between cardiovascular risk factors and participation in LTPA in a population of older adults with long-term SCI. Our hypothesis was partly supported as more minutes per day of LTPA of at least moderate intensity were significantly associated with a lower BMI and a lower WC, after adjusting for sociodemographics, injury characteristics and tobacco use. In addition, more minutes per day of LTPA of all intensities were significantly associated with a higher systolic BP among participants with tetraplegia.

\section{Associations between cardiovascular risk factors and leisure time physical activity}

\section{Body mass index and waist circumference}

The regression analyses revealed that more minutes per day of moderate-to-heavy LTPA were significantly associated with a lower BMI and WC. These results are supported by previous cross-sectional research using the PARA-SCI $(n=$ 56 , mean age 42 years, mean time since injury 14.5 years, 79\% men) [12]. However, a more recent Swedish study could not confirm this finding $(n=134$, mean age 48 years, mean time since injury 18.5 years, $77 \%$ men) [20]. The latter study included only participants with wheelchairdependent paraplegia, which may explain the differences between their findings and our results. The most frequently 
Table 3 Multivariable linear regression models investigating the association between body mass index, waist circumference and moderate-to-heavy leisure time physical activity among older adults with long-term spinal cord injury, adjusted for sociodemographics, injury characteristics and tobacco use

\begin{tabular}{|c|c|c|c|c|c|c|}
\hline \multirow[t]{2}{*}{ Independent variables } & \multicolumn{3}{|c|}{ Body mass index $(n=117)$} & \multicolumn{3}{|c|}{ Waist circumference $(n=115)$} \\
\hline & Beta & B & $95 \% \mathrm{CI}$ for $\mathrm{B}$ & Beta & B & $95 \% \mathrm{CI}$ for $\mathrm{B}$ \\
\hline $\begin{array}{l}\text { Moderate-to-heavy LTPA; min/ } \\
\text { day }\end{array}$ & $-0.31 * *$ & $-0.04 * *$ & $-0.06,-0.02$ & $-0.24 * *$ & $-0.09 * *$ & $-0.16,-0.02$ \\
\hline Age; years & 0.05 & 0.02 & $-0.07,0.12$ & 0.07 & 0.10 & $-0.18,0.38$ \\
\hline Time since injury; years & -0.002 & -0.001 & $-0.07,0.07$ & 0.07 & 0.08 & $-0.14,0.29$ \\
\hline Female gender & -0.04 & -0.35 & $-2,18,1.48$ & $-0.24 *$ & $-6.68 *$ & $\begin{array}{l}-12.07 \\
-1.30\end{array}$ \\
\hline Non-traumatic injury & 0.16 & 1.46 & $-0.36,3.29$ & 0.07 & 1.81 & $-3.57,7.19$ \\
\hline Tetraplegia AIS A-C ${ }^{\mathbf{a}}$ & -0.08 & -0.98 & $-3.35,1.39$ & 0.18 & 6.14 & $-0.77,13.06$ \\
\hline Paraplegia AIS A-C & $0.24 *$ & $2.19^{*}$ & $0.28,4.09$ & $0.21 *$ & $5.84 *$ & $0.16,11.51$ \\
\hline Current daily smoking & -0.02 & -0.26 & $-2.59,2.07$ & -0.08 & -2.72 & $-9.40,3.96$ \\
\hline Adjusted $R^{2}$ & 0.11 & & & 0.13 & & \\
\hline
\end{tabular}

Beta standardized regression coefficient, $B$ unstandardized regression coefficient, $C I$ confidence interval, LTPA leisure time physical activity

$* p<0.05 ; * * p<0.01$

${ }^{a}$ Reference category: All AIS D reported activity in the SASCIS sample was walking and wheelchair use was the strongest explanatory factor for less participation in LTPA [17]. Our inclusion of participants with preserved ability to walk might therefore have contributed to a larger variability in LTPA participation, and thereby a detectable relationship between BMI and LTPA.

Considering the association between BMI and LTPA, it was not surprising that we also found a significant negative association between WC and moderate-to-heavy LTPA. Our results are again supported by previous findings [12]. In addition, a recent study by Pelletier et al. [27] ( $n=136$, mean age 49 years, mean time since injury 16 years, $74 \%$ men) showed a significant relationship between less visceral adipose tissue and moderate-to-heavy LTPA. Together, these findings infer that participation in LTPA has a promising effect on body composition, although causal inferences cannot be made due to the cross-sectional study designs.

Notably, only moderate-to-heavy LTPA contributed to the explained variance in BMI and WC. The results are well in line with the recently developed SCI-specific exercise guidelines, stating that at least moderate intensity physical activity is required for health benefits [14]. Controlled trials targeting older adults with long-term SCI are needed to establish the cause and effect of the associations found, and to extend the exercise guidelines into this segment of the SCI population. Nevertheless, our findings suggest that moderate-to-heavy LTPA may be included in interventions to decrease overweight and central adiposity also among older adults with long-term SCI, and thereby possibly reduce their risk of developing CVD.

Our independent variables, including moderate-to-heavy LTPA, explained less than $15 \%$ of the variance in BMI and WC. This indicates that several other factors can contribute to these risk markers of CVD among older adults with long-term SCI. For example, there is evidence of a relationship between cardiovascular health and socioeconomic and psychological factors, both in the general population and after SCI [28-31]. Whether these associations hold true also among older adults with long-term injury is yet to be determined.

\section{Blood pressure, lipid profiles and fasting plasma glucose}

LTPA did not exhibit any significant associations with BP when participants with different level and severity of injury were analyzed together. However, when separating the two main injury groups (tetraplegia and paraplegia), we found a significant positive relationship between total LTPA and systolic BP among participants with cervical injuries. This is in agreement with previous studies, which indicate a trend towards a higher systolic BP among active persons with tetraplegia as compared to inactive [12], as well as an increase in resting systolic BP after exercise among persons with tetraplegia [32, 33]. Taken together, these findings suggest that LTPA could be beneficial for persons with cervical lesions and hypotension. On the contrary, we could not confirm previous findings of a relationship between more LTPA and a lower BP among persons with paraplegia $[12,20]$. It is possible that our participants with paraplegia did not reach the amount of LTPA required to elicit an association with BP.

Moreover, we did not find any significant associations between blood lipids, FPG and LTPA. This is typically described in the non-injured population $[9,10]$, but our findings are, in fact, consistent with earlier research on selfreported LTPA and cardiovascular risk after SCI $[12,20]$. The lack of significant relationships does not necessarily mean that physical activity is unrelated to these risk 
indicators among older adults with long-term SCI. It might instead reflect that our participants performed several different types of LTPA of different intensities. In the literature on LTPA conceptualized as exercise, moderate-to-heavy intensity aerobic training exhibits a positive effect on blood lipid profiles and glucose metabolism after SCI [13, 34]. Considering the overall low participation in aerobic exercise among our participants and the variability in performed activities [17], it is likely that they did not reach the amount of aerobic training required for changes in blood lipids and glucose metabolism. Indeed, at least $30 \mathrm{~min}$ of moderate-toheavy aerobic exercise three times per week is required for positive effects on these parameters [14]. As half of the SASCIS sample performed less than 5 min of moderate-toheavy LTPA per day, the majority of our participants do not even come close to fulfilling these recommendations. Thus, the present study further adds to the contention that research on how to promote participation in LTPA among older adults with long-term SCI is of great importance to support a healthy and active aging after injury $[16,17]$.

\section{Strengths and limitations}

The studies in the SASCIS are conducted on data from a population-based, representative sample of older adults with long-term SCI in southern Sweden [21]. Furthermore, we used a SCI-specific questionnaire - the PARA-SCI - that has sound psychometric properties [25, 35], to assess LTPA. Self-report measures of physical activity are always susceptible to recall and response bias, but the PARA-SCI was recently suggested as the most suitable self-report assessment of LTPA in persons with physical disabilities [36]. The PARA-SCI has a short recall time (i.e., 3 days) which limits the risks for recall bias, and the SCI-specific intensity chart [25] facilitates accurate rating of activity intensity. There are also a few limitations to the present study. We included persons with various injury levels and AIS grades which could have concealed between-group differences in the associations between the cardiovascular risk factors and LTPA. However, the sample size did not permit further separate analyses according to the level and severity of injury. Due to the cross-sectional study design, we were not able to identify causal relationships between variables.

\section{Conclusion}

The present study suggests an association between cardiovascular health and participation in daily moderate-to-heavy LTPA among older adults with long-term SCI, where both BMI and WC decrease with increasing LTPA. In addition, systolic BP increases with more LTPA of all intensities, but only among participants with tetraplegia. This study can serve as a starting point for future research to establish the directional causality of the associations found, and to determine the amount of activity needed to obtain positive health effects among older adults with long-term SCI.

\section{Data archiving}

All the data were archived according to the Swedish Act concerning the Ethical Review of Research Involving Humans to attain confidentiality and are available from the corresponding author upon reasonable request.

Acknowledgements We are grateful to all participants. We also wish to thank Lizette Norin, BSc, Department of Health Sciences, Lund University, for assistance during the data collection.

Funding: This study was carried out within the framework of the Swedish Aging with Spinal Cord Injury Study (SASCIS), financed by research grants from the Swedish Research Council, the NorrbackaEugenia Foundation, the Promobilia Foundation, the Research Fund of Neuro Sweden, the Swedish Association for Survivors of Accident and Injury (RTP), Gun and Bertil Stohne's Foundation, the Ribbingska Foundation in Lund, and the Gustaf V and Queen Victoria's Freemason Foundation. The baseline data collection in the SASCIS was accomplished within the context of the Centre for Ageing and Supportive Environments (CASE), Lund University, financed by the Swedish Research Council for Health, Working Life and Welfare.

Author contributions: The SASCIS was initiated and designed by SJ and JL. SJ, KMG and JL designed the present study, SJ performed the data analyses and drafted the manuscript in collaboration with SS, LM and JL. All authors critically revised the manuscript and all authors have read and approved the final version of the manuscript.

\section{Compliance with ethical standards}

Conflict of interest The authors declare that they have no conflict of interest.

Ethical approval The principles of the Declaration of Helsinki for research on humans were followed and the SASCIS was approved by the Regional Ethical Review Board in Lund, Sweden (number 2010/ 692). All participants were given written and oral information about the study before providing their written informed consent to participate. We certify that all applicable institutional and governmental regulations concerning the ethical use of human volunteers were followed during the course of this research.

Publisher's note: Springer Nature remains neutral with regard to jurisdictional claims in published maps and institutional affiliations.

\section{References}

1. Warburton DER, Krassioukov A, Sproule S, Eng JJ. Cardiovascular health and exercise following spinal cord injury. In: Eng JJ, Teasell RW, Miller WC, Wolfe DL, Townson AF, Hsieh JTC, et al., editors. Spinal cord injury rehabilitation evidence, version 5. 2014. p. 1-48. 
https://scireproject.com/evidence/rehabilitation-evidence/cardiova scular-health-and-exercise/. Accessed August 15, 2018.

2. Wahman K, Nash MS, Lewis JE, Seiger Å, Levi R. Increased cardiovascular disease risk in Swedish persons with paraplegia: The Stockholm spinal cord injury study. J Rehabil Med. 2010;42:489-92.

3. Myers J, Lee M, Kiratli J. Cardiovascular disease in spinal cord injury: an overview of prevalence, risk, evaluation, and management. Am J Phys Med Rehabil. 2007;86:142-52.

4. Savic G, Charlifue S. Aging with spinal cord injury. In: Chhabra HS, editor. ISCoS textbook on comprehensive management of spinal cord injuries.1st ed, Haryana, India: Lippincott Williams \& Wilkins; 2015. p. 906-17.

5. Garshick E, Kelley A, Cohen S, Garrison A, Tun CG, Gagnon D, et al. A prospective assessment of mortality in chronic spinal cord injury. Spinal Cord. 2005;43:408-16.

6. Savic G, DeVivo MJ, Frankel HL, Jamous MA, Soni BM, Charlifue S. Causes of death after traumatic spinal cord injury-a 70-year British study. Spinal Cord. 2017;55:891-7.

7. Jensen MP, Truitt AR, Schomer KG, Yorkston KM, Baylor C, Molton IR. Frequency and age effects of secondary health conditions in individuals with spinal cord injury: a scoping review. Spinal Cord. 2013;51:882-92.

8. Groah SL, Weitzenkamp D, Sett P, Soni B, Savic G. The relationship between neurological level of injury and symptomatic cardiovascular disease risk in the aging spinal injured. Spinal Cord. 2001;39:310-7.

9. World Health Organization. Global recommendations on physical activity for health. Geneva, Switzerland: World Health Organization; 2010.

10. Catapano AL, Graham I, De Backer G, Wiklund O, Chapman MJ, Drexel H, et al. 2016 ESC/EAS Guidelines for the Management of Dyslipidaemias. Eur Heart J. 2016;37:2999-3058.

11. Martin Ginis KA, Latimer AE, Buchholz AC, Bray SR, Craven $\mathrm{BC}$, Hayes $\mathrm{KC}$, et al. Establishing evidence-based physical activity guidelines: methods for the Study of Health and Activity in People with Spinal Cord Injury (SHAPE SCI). Spinal Cord. 2008;46:216-21.

12. Buchholz AC, Martin Ginis KA, Bray SR, Craven BC, Hicks AL, Hayes KC, et al. Greater daily leisure time physical activity is associated with lower chronic disease risk in adults with spinal cord injury. Appl Physiol Nutr Metab. 2009;34:640-7.

13. van der Scheer JW, Martin Ginis KA, Ditor DS, Goosey-Tolfrey VL, Hicks AL, West CR, et al. Effects of exercise on fitness and health of adults with spinal cord injury: a systematic review. Neurology. 2017;89:736-45.

14. Martin Ginis KA, van der Scheer JW, Latimer-Cheung AE, Barrow A, Bourne C, Carruthers $\mathrm{P}$, et al. Evidence-based scientific exercise guidelines for adults with spinal cord injury: an update and a new guideline. Spinal Cord. 2018;56:308-21.

15. Groah SL, Charlifue S, Tate D, Jensen MP, Molton IR, Forchheimer M, et al. Spinal cord injury and aging: challenges and recommendations for future research. Am J Phys Med Rehabil. 2012;91:80-93.

16. Jörgensen S. Older adults with long-term spinal cord injury [dissertation]. Lund: Lund University; 2017.

17. Jörgensen S, Martin Ginis KA, Lexell J. Leisure time physical activity among older adults with long-term spinal cord injury. Spinal Cord. 2017;55:848-56.

18. Martin Ginis KA, Latimer AE, Arbour-Nicitopoulos KP, Buchholz AC, Bray SR, Craven BC, et al. Leisure time physical activity in a population-based sample of people with spinal cord injury part I: demographic and injury-related correlates. Arch Phys Med Rehabil. 2010;91:722-8.
19. Jörgensen S, Hill M, Lexell J. Cardiovascular risk factors among older adults with long-term spinal cord injury. PM R. 2018. https://doi.org/10.1016/j.pmrj.2018.06.008.

20. Flank P, Fahlström M, Boström C, Lewis JE, Levi R, Wahman K. Self-reported physical activity and risk markers for cardiovascular disease after spinal cord injury. J Rehabil Med. 2014;46:886-90.

21. Jörgensen S, Iwarsson S, Norin L, Lexell J. The Swedish Aging with Spinal Cord Injury Study (SASCIS): methodology and initial results. PM R. 2016;8:667-77.

22. Jörgensen S, Iwarsson S, Lexell J. Secondary health conditions, activity limitations, and life satisfaction in older adults with longterm spinal cord injury. PM R. 2017;9:356-66.

23. Jörgensen S, Martin Ginis KA, Iwarsson S, Lexell J. Depressive symptoms among older adults with long-term spinal cord injury: Associations with secondary health conditions, sense of coherence, coping strategies and physical activity. J Rehabil Med. 2017;49:644-51.

24. Kirshblum SC, Burns SP, Biering-Sorensen F, Donovan W, Graves DE, Jha A, et al. International standards for neurological classification of spinal cord injury (revised 2011). J Spinal Cord Med. 2011;34:535-46.

25. Martin Ginis KA, Latimer AE, Hicks AL, Craven BC. Development and evaluation of an activity measure for people with spinal cord injury. Med Sci Sports Exerc. 2005;37:1099-111.

26. Martin Ginis KA, Latimer AE. Physical activity recall assessment for people with spinal cord injury: administration and scoring manual. Hamilton, Ontario, Canada: McMaster University; 2008.

27. Pelletier CA, Omidvar M, Miyatani M, Giangregorio L, Craven BC. Participation in moderate-to-vigorous leisure time physical activity is related to decreased visceral adipose tissue in adults with spinal cord injury. Appl Physiol Nutr Metab. 2018;43:139-44.

28. Psaltopoulou T, Hatzis G, Papageorgiou N, Androulakis E, Briasoulis A, Tousoulis D. Socioeconomic status and risk factors for cardiovascular disease: Impact of dietary mediators. Hell $\mathbf{J}$ Cardiol. 2017;58:32-42.

29. Krause JS, Cao Y, DeVivo MJ, DiPiro ND. Risk and protective factors for cause-specific mortality after spinal cord injury. Arch Phys Med Rehabil. 2016;97:1669-78.

30. Krause JS, Carter RE, Pickelsimer EE, Wilson D. A prospective study of health and risk of mortality after spinal cord injury. Arch Phys Med Rehabil. 2008;89:1482-91.

31. Eriksson M, Lindström B. Antonovsky's sense of coherence scale and the relation with health: a systematic review. J Epidemiol Community Health. 2006;60:376-81.

32. Carvalho DC, Cliquet A, Jr. Response of the arterial blood pressure of quadriplegic patients to treadmill gait training. Braz J Med Biol Res. 2005;38:1367-73.

33. Faghri PD, Glaser RM, Figoni SF. Functional electrical stimulation leg cycle ergometer exercise: training effects on cardiorespiratory responses of spinal cord injured subjects at rest and during submaximal exercise. Arch Phys Med Rehabil. 1992;73:1085-93.

34. Bresnahan JJ, Farkas GJ, Clasey JL, Yates JW, Gater DR. Arm crank ergometry improves cardiovascular disease risk factors and community mobility independent of body composition in high motor complete spinal cord injury. J Spinal Cord Med. 2018. https://doi.org/10.1080/10790268.2017.1412562.

35. Latimer AE, Martin Ginis KA, Craven BC, Hicks AL. The physical activity recall assessment for people with spinal cord injury: validity. Med Sci Sports Exerc. 2006;38:208-16.

36. Nightingale TE, Rouse PC, Thompson D, Bilzon JLJ. Measurement of physical activity and energy expenditure in wheelchair users: Methods, considerations and future directions. Sports Med Open. 2017;3:10. 